\title{
Microwave response of a cylindrical cavity made of bulk $\mathrm{MgB}_{2}$ superconductor
}

\author{
A. Agliolo Gallitto ${ }^{\text {a }}$, G. Bonsignore ${ }^{\text {a }}$, M. Li Vigni ${ }^{\mathrm{a}, *}$, \\ G. Giunchi ${ }^{\text {b }}$, Yu. A. Nefyodov ${ }^{\mathrm{c}}$ \\ ${ }^{a}$ CNISM and Dipartimento di Scienze Fisiche e Astronomiche, Università di \\ Palermo, via Archirafi 36, 90123 Palermo, Italy \\ ${ }^{\mathrm{b}}$ EDISON S.p.A, Divisione Ricerca e Sviluppo, foro Buonaparte 31, 20121 Milano, \\ Italy \\ ${ }^{\mathrm{c}}$ Institute of Solid State Physics, Chernogolovka, Moscow District 142432, Russia
}

\begin{abstract}
We report on the microwave properties of a resonant cylindrical cavity made of bulk $\mathrm{MgB}_{2}$ superconductor, produced by the reactive liquid $\mathrm{Mg}$ infiltration process. The frequency response of the cavity has been measured in the range $5 \div 13 \mathrm{GHz}$. Among the various modes, the $\mathrm{TE}_{011}$, resonating at $9.79 \mathrm{GHz}$, exhibits the highest quality factor. For this mode, we have determined the temperature dependence of the quality factor, obtaining values of the order of $10^{5}$ in the temperature range $4.2 \div 30 \mathrm{~K}$. The values of the surface resistance deduced from the measurements of the quality factor agree quite well with those independently measured in a small sample of $\mathrm{MgB}_{2}$ extracted from the same specimen from which the cavity has been obtained.
\end{abstract}

Key words: Superconducting microwave cavity, $\mathrm{MgB}_{2}$, Surface resistance PACS: 85.25.Am, 74.25.Nf, 74.70.Ad

\section{Introduction}

Superconducting materials, because of their low surface resistance, are recommended for manufacturing many passive $m w$ devices [1,2]. Among the various devices, the superconducting resonant cavity is one of the most important application in the systems requiring high selectivity in the signal frequency, such

* Tel.:+39 0916234208; fax: +39 0916162461; e-mail: livigni@fisica.unipa.it 
as filters for communication systems [3], particle accelerators [4,5], equipment for material characterization at $m w$ frequencies [6,7].

Since the discovery of the high-temperature superconductors (HTS), several attempts have been done to assemble $m w$ cavities made of bulk HTS [3,6,8]; however, limitations in their performance were encountered. Firstly, grain boundaries in these materials are weakly coupled giving rise to reduction of the critical current and/or nonlinear effects, which worsen the device performance [9]; furthermore, the process necessary to obtain bulk HTS in a performing textured form is very elaborated. For these reasons, in several applications, most of the superconducting cavities are still manufactured with $\mathrm{Nb}$, requiring liquid $\mathrm{He}$ as refrigerator [4,7, 10].

Since the discovery of superconductivity at $39 \mathrm{~K}$ in $\mathrm{MgB}_{2}$ [1], several authors have indicated this material as promising for technological applications [5, 12,13]. The advantages of using $\mathrm{MgB}_{2}$ for this purpose are several. The simple chemical composition makes the synthesis process easy, reducing the production cost in comparison with HTS. The relatively high critical temperature allows to maintain the compound in the superconducting state by using modern closed-cycle cryocoolers, not requiring liquid He as thermal bath. A further interesting property of $\mathrm{MgB}_{2}$ bulk samples is the high connectivity of the superconducting grains. Indeed, contrary to oxide HTS, bulk $\mathrm{MgB}_{2}$ can be used in the polycrystalline form without a significant degradation of its critical current [12:13 14]. This property has been ascribed to the large coherence length, which makes the material less susceptible to structural defects like grain boundaries [15. Due to these amazing properties, $\mathrm{MgB}_{2}$ has been recommended for manufacturing $m w$ cavities [5,16], and investigation is carried out to test the potential of different $\mathrm{MgB}_{2}$ materials for this purpose.

In this paper, we report on the $m w$ properties of the first cylindrical cavity made of bulk $\mathrm{MgB}_{2}$ [17]. The material has been produced by the reactive liquid $\mathrm{Mg}$ infiltration technology [1819; it exhibits a critical temperature $T_{c} \approx 38.5 \mathrm{~K}$. The frequency response of the cavity has been measured in the range $5 \div 13 \mathrm{GHz}$. The quality factor of the cavity for the $\mathrm{TE}_{011}$ mode has been investigated as a function of the temperature, from $T=4.2 \mathrm{~K}$ up to $T \approx 150 \mathrm{~K}$. From the $Q$ values, we have determined the temperature dependence of the $m w$ surface resistance of the $\mathrm{MgB}_{2}$ material; these results have been compared with those independently obtained in a small plate-like sample of $\mathrm{MgB}_{2}$ extracted from the same specimen used for the cavity. 


\section{Cylindrical cavities: theoretical aspects}

As it is well known, an important valuation index to determine the performance of a resonant cavity is the quality factor $Q$, defined as

$$
Q=2 \pi \frac{\text { energy stored in the cavity }}{\text { energy lost per cycle }} \text {. }
$$

In an empty resonant cavity, essentially two causes contribute to the energy losses: conductor losses, associated to the conduction currents in the cavity walls; radiation losses, due to power that leaves the cavity through the holes for the coupling between the resonator and the external circuit. Therefore, the overall or loaded $Q$, denoted by $Q^{L}$, can be defined by

$$
\frac{1}{Q^{L}}=\frac{1}{Q^{U}}+\frac{1}{Q^{R}}
$$

where $Q^{R}$ is due to the radiative losses and $Q^{U}$, the so-called unloaded $Q$, includes only the conductor losses.

$Q^{U}$ is given by

$$
Q^{U}=\frac{\Gamma}{R_{s}}
$$

where $R_{s}$ is the surface resistance of the material; $\Gamma$ is the geometry factor, it depends on the shape and dimensions of the cavity and the specific resonant mode.

For a cavity coupled to the external circuit through two lines, $Q^{U}$ can be deduced, from the measured $Q^{L}$, by taking into account the coupling coefficients, $\beta_{1}$ and $\beta_{2}$, for both the coupling lines; these coefficients can be calculated by directly measuring the reflected power at each line, as described in Chap. IV of Ref. [1]. Thus, $Q^{U}$ can be calculated as

$$
Q^{U}=\left(1+\beta_{1}+\beta_{2}\right) Q^{L}
$$

It is worth noting that $Q^{R}$ can be made large by weakly coupling the excitation and detection lines. In this case, $\beta_{1}+\beta_{2} \ll 1$ and $Q^{U} \approx Q^{L}$.

As it is well known, resonant cylindrical cavities can support both $\mathrm{TE}_{l m n}$ and $\mathrm{TM}_{l m n}$ modes; the subscripts $l, m$ and $n$ refer to the number of half-wavelength variations in the standing-wave pattern in $\phi, r$ and $z$ directions, respectively. The resonant frequencies of a cylindrical cavity resonating in the $\mathrm{TE}_{l m n}$ or $\mathrm{TM}_{l m n}$ modes are given by [1]

$$
f_{l m n}^{T E}=\frac{1}{2 \pi \sqrt{\epsilon \mu}} \sqrt{\left(\frac{n \pi}{d}\right)^{2}+\left(\frac{z_{l m}^{\prime}}{a}\right)^{2}}
$$




$$
f_{l m n}^{T M}=\frac{1}{2 \pi \sqrt{\epsilon \mu}} \sqrt{\left(\frac{n \pi}{d}\right)^{2}+\left(\frac{z_{l m}}{a}\right)^{2}},
$$

where $\mu$ and $\epsilon$ are the permeability and dielectric constant of the medium filling the cavity; $a$ and $d$ the radius and length of the cavity; $z_{l m}$ and $z_{l m}^{\prime}$ are the $m$ th zeros of the Bessel function of order $l$ and of its first derivative, respectively.

Among the various resonant modes, the ones more extensively used are the $\mathrm{TE}_{01 n}$, which give a reasonably high quality factor. In the $\mathrm{TE}_{01 n}$ modes, the wall currents are purely circumferential and no currents flow between the end plates and the cylinder. This implies that no electrical contact between plates and cylinder is required, making the cavity assembly easy. The $\mathrm{TE}_{01 n}$ modes are degenerate in frequency with the $\mathrm{TM}_{11 n}$ modes and this should be avoided to have a well defined field configuration; however, this degeneracy can be removed introducing a small gap between cylinder and lids. This shifts the resonant frequency of the $\mathrm{TM}_{11 n}$ modes downwards, leaving the $\mathrm{TE}_{01 n}$ modes nearly unperturbed. The unloaded quality factor of a cylindrical cavity resonating in the $\mathrm{TE}_{01 n}$ mode is given by

$$
Q_{01 n}^{U}=\frac{\Gamma_{01 n}}{R_{s}}
$$

with

$$
\Gamma_{01 n}=\sqrt{\frac{\mu}{\epsilon}} \frac{\left[\left(z_{01}^{\prime} d\right)^{2}+(n \pi a)^{2}\right]^{3 / 2}}{2\left(z_{01}^{\prime}\right)^{2} d^{3}+4 n^{2} \pi^{2} a^{3}}, \quad z_{01}^{\prime}=3.83170
$$

\section{The superconducting $\mathrm{MgB}_{2}$ cavity}

The material from which the cavity is made has been produced by the reactive liquid $\mathrm{MgB}_{2}$ infiltration technology (RLI) [18,19]. The RLI process consists in the reaction of pure liquid $\mathrm{Mg}$ and boron powder, sealed in a stainless steel container with a weight ratio $\mathrm{Mg} / \mathrm{B}$ over the stoichiometric value $(\approx 0.55)$. The reaction between liquid $\mathrm{Mg}$ and $\mathrm{B}$ powder produces a more stable material, with respect to the hot pressing technique, at temperatures of a few hundred $\mathrm{K}$ above the Mg melting point and at moderate pressure. This procedure allows one to obtain specimens of high density and dimensions of the order of tens on centimeters, showing very high mechanical strength [19,20].

As the first attempt to apply the $\mathrm{MgB}_{2}$ produced by RLI to the cavity-filter technology, we have manufactured a simple cylindrical cavity and have investigated its microwave response in a wide range of frequencies. All the parts of the cavity, cylinder and lids, are made of bulk $\mathrm{MgB}_{2}$ material with $T_{c} \approx 38.5 \mathrm{~K}$ and density $\approx 2.33 \mathrm{~g} / \mathrm{cm}^{3}$. The material has been obtained using commercial 


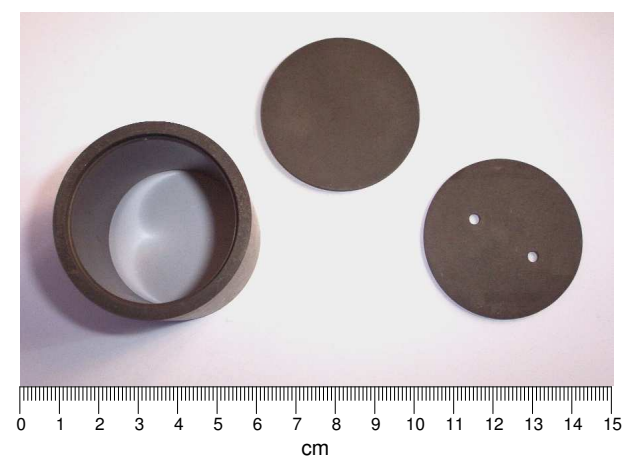

Fig. 1. Photograph of the cylinder and the lids composing the bulk $\mathrm{MgB}_{2}$ cavity. The holes in one of the lids are used to insert the coupling loops. After Ref.[17].

crystalline B powder (Starck H. C., Germany, 99.5\% purity) and pure Mg; in order to get the B powder to be used in the synthesis process, the original chunks were mechanically crushed and filtered through a 100 micron sieve. In particular, the present cylindrical cavity (inner diameter $40 \mathrm{~mm}$, outer diameter $48 \mathrm{~mm}$, height $42.5 \mathrm{~mm}$ ) was cut by electroerosion from a thicker bulk $\mathrm{MgB}_{2}$ cylinder, previously prepared as described in Sec. 4.3 of Ref. [20], internally polished to a surface roughness of about $300 \mathrm{~nm}$.

A photograph of the parts, cylinder and lids, composing the superconducting cavity is shown in Fig. 3. The holes in one of the lids have been used to insert two small loop antennas which couple the cavity with the excitation and detection lines. The loop antenna was constructed on the end of each line, soldering the central conductor to the outer shielding of the semirigid cable. In order to remove the degeneracy between $\mathrm{TE}_{01 n}$ and $\mathrm{TM}_{11 n}$ modes, we have incorporated a "mode trap" in the form of circular grooves $(1 \mathrm{~mm}$ thick, $2 \mathrm{~mm}$ wide) inside the cylinder at the outer edges.

\section{Experimental results}

The resonant cavity has been characterized by measuring its frequency response in the range $5 \div 13 \mathrm{GHz}$ by an $h p$-8719D Network Analyzer, using a two-port configuration. The spectrum of the cavity, measured at $T=4.2 \mathrm{~K}$, is shown in Fig. 2, in four frequency intervals. The arrows indicate the resonances we have certainly recognized by Eqs. (5) and (6); some peaks show a not simple structure, probably due to the superposition of different modes, which hinders to recognize the corresponding mode.

Among the various modes detected, two of them show the highest quality factors; at $T=4.2 \mathrm{~K}$, with the cavity filled by liquid He, the resonant frequencies 


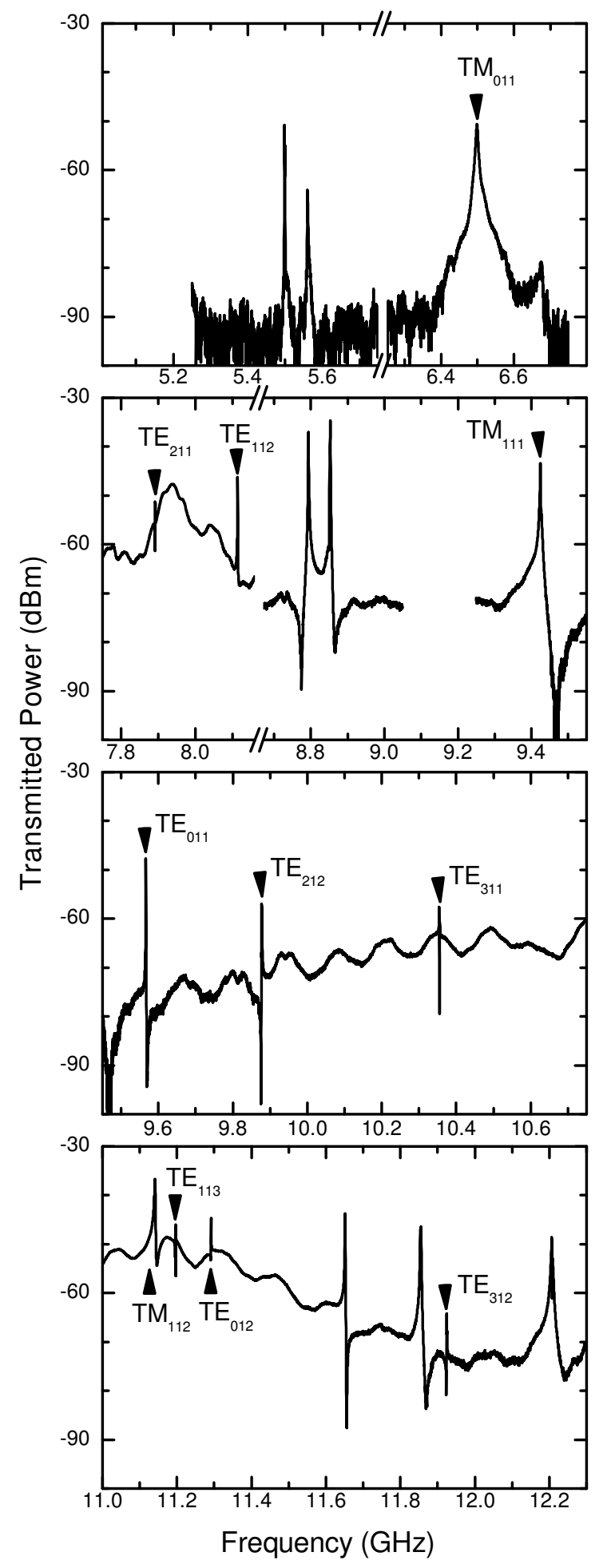

Fig. 2. Microwave response of the $\mathrm{MgB}_{2}$ cavity filled by liquid He in the frequency range $5.3 \div 12.3 \mathrm{GHz}$. In the omitted ranges, no resonant curves were present.

of these modes are $9.567 \mathrm{GHz}$ and $11.291 \mathrm{GHz}$. At room temperature, with the cavity filled by gaseous $\mathrm{He}$, these resonant frequencies move to $9.79 \mathrm{GHz}$ and $11.54 \mathrm{GHz}$; according to Eq. (5) , they correspond to the $\mathrm{TE}_{011}$ and $\mathrm{TE}_{012}$ 


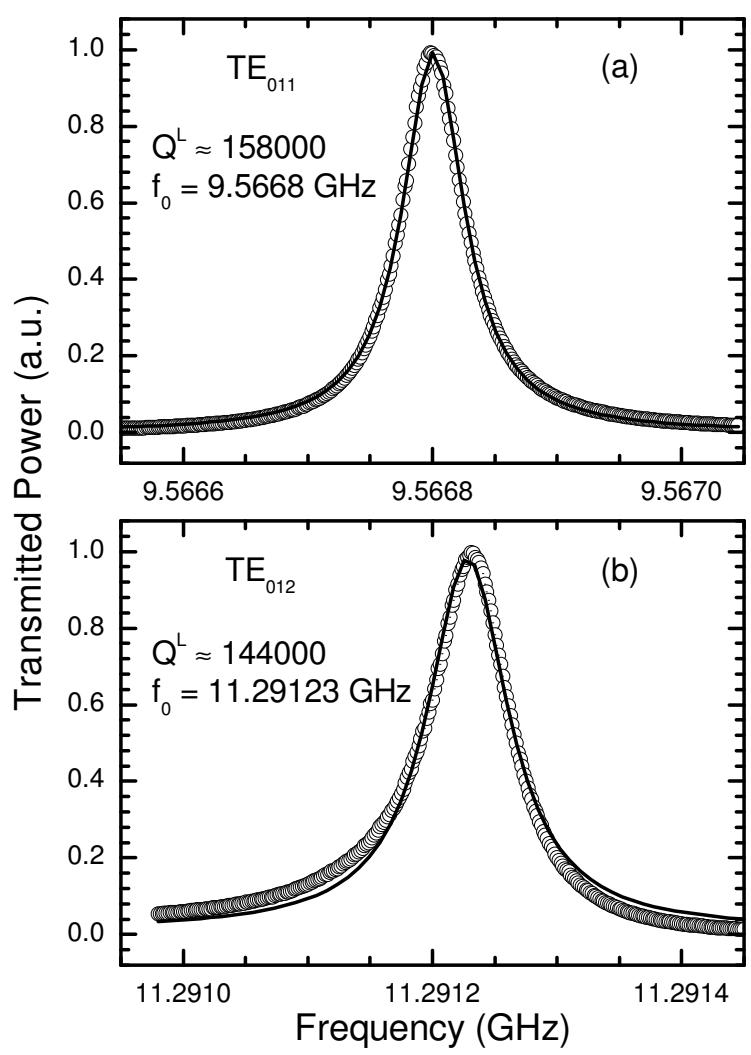

Fig. 3. Resonant curves, at $T=4.2 \mathrm{~K}$, corresponding to the $\mathrm{TE}_{011}$ (a) and $\mathrm{TE}_{012}$ (b) modes. The lines are Lorentzian fits of the experimental curves from which we have extracted the values of $Q^{L}$ and the central frequency $f_{0}$.

modes. Fig. 3 shows the resonant curves, at $T=4.2 \mathrm{~K}$, corresponding to the $\mathrm{TE}_{011}$ (a) and $\mathrm{TE}_{012}$ (b) modes. The lines are Lorentzian fits of the experimental data, which give for the loaded quality factors $Q_{011}^{L} \approx 158000$ and $Q_{012}^{L} \approx 144000$.

The quality factor of the $\mathrm{TE}_{011}$ mode has been measured as a function of the temperature, in the range $4.2 \div 150 \mathrm{~K}$. In order to calculate the unloaded $Q$ values, we have determined the coupling coefficients by measuring the power reflected by each line. The coupling coefficients, $\beta_{1}$ and $\beta_{2}$, have been measured at different values of the temperature; they result $\approx 0.2$ at $T=4.2 \mathrm{~K}$ and reduce to $\approx 0.05$ when the material goes to the normal state, at $T \approx 38.5 \mathrm{~K}$. Fig. 4 shows the temperature dependence of the loaded and unloaded $Q$ for the TE 011 mode, $Q_{011}^{L}$ and $Q_{011}^{U}$. At $T=4.2 \mathrm{~K}$, we have obtained $Q_{011}^{U}=2.2 \times 10^{5}$; on increasing the temperature, the quality factor maintains values of the order of $10^{5}$ up to $T \approx 30 \mathrm{~K}$ and reduces by a factor of $\approx 20$ at $T=T_{c}$. From the values of $Q_{011}^{U}$, it is possible, using Eq. (7), to deduce the surface resistance of the $\mathrm{MgB}_{2}$ material from which the cavity is made. In order to identify possible spurious effects in the measurement of the $m w$ response of the cavity, we have measured the $m w$ surface resistance of a small plate-like sample of $\mathrm{MgB}_{2}$ extracted from the same specimen from which the cavity has been obtained. 


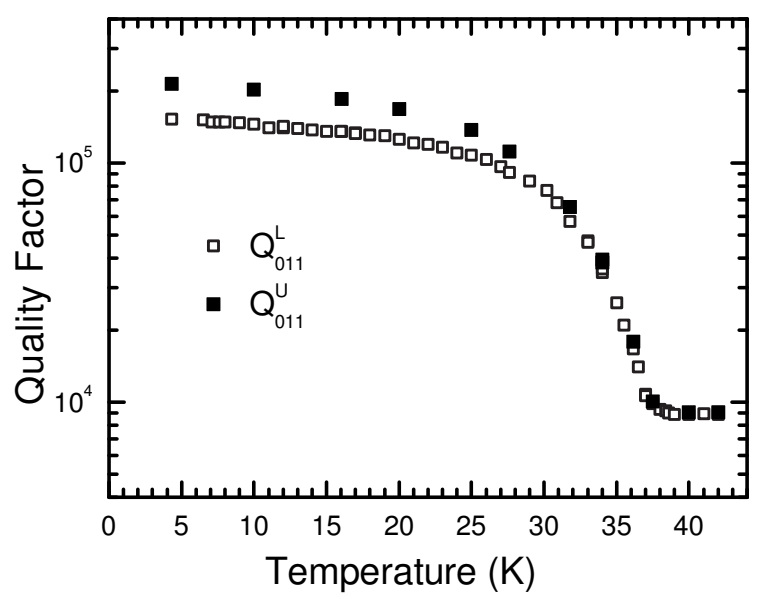

Fig. 4. Temperature dependence of the loaded- and unloaded- $Q$ values, $Q_{011}^{L}$ and $Q_{011}^{U}$, for the $\mathrm{TE}_{011}$ mode.

These measurements have been performed by the technique of hot-finger cavity perturbation [10], using a $\mathrm{Nb}$ cavity resonating at $\approx 9.4 \mathrm{GHz}$. Fig. 5 shows a comparison between the $R_{s}(T)$ values deduced from $Q_{011}^{U}(T)$ (full symbols) and those obtained in the sample (open symbols). As one can see, when the cavity is in the superconducting state, the $R_{s}(T)$ curves obtained by the two different techniques are consistent, while for $T>T_{c}$ they differ by $\approx 10 \%$. This disagreement may be ascribed to the difficulty of measuring with high sensitivity the quality factor of the $\mathrm{MgB}_{2}$ cavity at $T>T_{c}$, due to its small value. On the contrary, the sensitivity of the measurements performed by the technique of hot-finger cavity perturbation is very high independently of the temperature; indeed, the $\mathrm{Nb}$ cavity is always maintained in the superconducting state and only the temperature of the sample is changed.

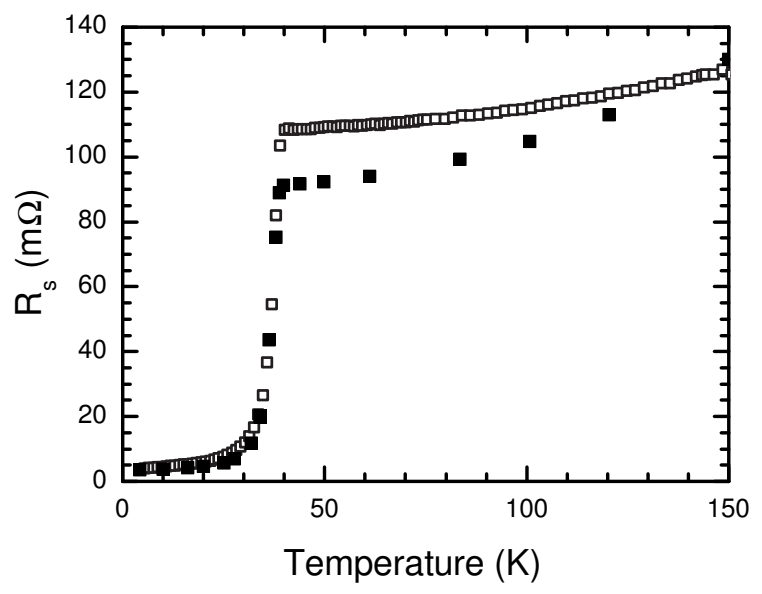

Fig. 5. Comparison between the temperature dependence of $R_{s}(T)$ deduced from $Q_{011}^{U}(T)$ (full symbols) and that obtained at $9.4 \mathrm{GHz}$ in a small $\mathrm{MgB}_{2}$ sample extracted from the same material of the cavity (open symbols).

As one can see from Fig. $3 \mathrm{~b}$, the resonant curve for the $\mathrm{TE}_{012}$ mode is not very 
well described by a Lorentzian function; this problem becomes more significant on increasing the temperature, making inaccurate the determination of the quality factor at temperatures higher that $\approx 30 \mathrm{~K}$. For these reason, we have not reported here the temperature dependence of $Q_{012}$. However, we have verified that, also for the $\mathrm{TE}_{102}$ mode, the quality factor takes on values of the order of $10^{5}$ up to $T \sim 30 \mathrm{~K}$ and reduced by a factor of $\approx 20$ when the material goes to the normal state.

\section{Discussion and Conclusion}

It is well known that one of the most important application of superconducting materials is the implementation of $m w$ resonant cavities, which can be used in a large variety of devices $[1,3,4,5,6,7]$. Although several studies have been devoted to build $m w$ cavities using HTS, actually most of the superconducting cavities are still manufactured with $\mathrm{Nb}$, requiring liquid He as refrigerator. The main problem encountered in using bulk HTS, for this purpose, is due to the weak coupling of grain boundaries in these materials, which act as weak links. On the contrary, it has widely been shown that in bulk $\mathrm{MgB}_{2}$ samples only a small amount of grain boundaries act as weak links [15,21,22,23, hindering the strong suppression of the critical current with magnetic field and the generation of nonlinear effects at high input power. For these reasons, as well as for the relatively high $T_{c}$, several authors have recommended $\mathrm{MgB}_{2}$ as a convenient material to build $m w$ cavity.

Recently, we have investigated the $m w$ response of small plate-like samples of $\mathrm{MgB}_{2}$ prepared by RLI process, in the linear and nonlinear regimes [23,24]. These studies have highlighted a weak nonlinear response, as well as relatively small values of the residual surface resistance. In particular, we have found that the $m w$ properties of such samples improve on decreasing the size of the B powder used in the synthesis process. Furthermore, bulk samples produced by RLI maintain the surface staining unchanged for years, without controlledatmosphere protection. This useful property is most likely related to the high density, and consequently high grain connectivity, achieved with the RLI process, as well as to the small and controlled amount of impurity phases [25]. Prompted by these interesting results, we have built the $m w$ resonant cavity using $\mathrm{MgB}_{2}$ produced by the RLI process [17].

The results of Fig. 4 show that $Q$ takes on values of the order of $10^{5}$ from $T=4.2 \mathrm{~K}$ up to $T \approx 30 \mathrm{~K}$, a temperature easily reachable by modern closedcycle cryocoolers. To our knowledge, these $Q$ values are higher than those reported in the literature for $m w$ cylindrical cavities manufactured with HTS, both bulk and films $3,6,8$. So, our results show that $\mathrm{MgB}_{2}$ produced by RLI is a very promising material for building $m w$ resonant cavities. We would 
remark that this is the first attempt to realize a superconducting cavity made of bulk $\mathrm{MgB}_{2}$. In particular, this investigation has been carried out with the aim to explore the potential of bulk $\mathrm{MgB}_{2}$ materials prepared by RLI to the mw-cavity technology.

The $\mathrm{MgB}_{2}$ material from which the present cavity is made has been obtained using crystalline $\mathrm{B}$ powder with grain mean size $\approx 100 \mu \mathrm{m}$. Our previous studies on bulk $\mathrm{MgB}_{2}$ samples, obtained by the RLI method, have shown that samples prepared using microcrystalline B powder $(\approx 1 \mu \mathrm{m}$ in size $)$ exhibit smaller residual surface resistance $(\approx 0.5 \mathrm{~m} \Omega)$ [24]. From Fig. 5 , one can see that the residual surface resistance of the cavity is $R_{s}(4.2 \mathrm{~K}) \approx 3.5 \mathrm{~m} \Omega$. So, we infer that one could improve the quality factor by one order of magnitude manufacturing the cavity with material produced by liquid $\mathrm{Mg}$ infiltration in micrometric B powder.

Because of the shorter length of percolation of the liquid $\mathrm{Mg}$ into very fine $\mathrm{B}$ powder, the production of massive $\mathrm{MgB}_{2}$ samples by RLI using $\mathrm{B}$ powder with size $\approx 1 \mu \mathrm{m}$ turns out to be more elaborate. However, work is in progress to improve the preparation process in order to manufacture large specimens using microcrystalline B powder. Considering that the $m w$ fields penetrate in a surface layer of the material of the order of the penetration depth, a more performing $m w$ resonant cavity can be obtained designing a composite structure; in particular, one can use microcrystalline B powder for the inner part of the cavity and larger grain-size B powder for the outer part.

In conclusion, we have shown that the RLI process provides a useful method for assembling high-performance $m w$ cavities, which may have large scale application. We have obtained quality factors of the order of $10^{5}$, larger than those reported in the literature for cavities made of HTS, both bulk and films, in the same temperature range. Although higher quality factors have been reported for superconducting cavities made of pure $\mathrm{Nb}$ and $\mathrm{Nb}$ alloys [4], $\mathrm{Nb}$ cavities must be kept in liquid-He bath; on the contrary, cavity made of $\mathrm{MgB}_{2}$ can be maintained in the superconducting state by using close-cycle cryocoolers that can easily work at temperature of the order of $10 \mathrm{~K}$.

\section{Acknowledgements}

The authors are very glad to thank M. Bonura for his continuous interest and helpful suggestions, G. Lapis and G. Napoli for technical assistance. Work partially supported by the University of Palermo (grant Coll. Int. Li Vigni, Co.RI 2005). 


\section{References}

[1] M. J. Lancaster, Passive Microwave Device Applications of High-Temperature Superconductors, Cambridge University Press (Cambridge 1997).

[2] M. Hein, High-Temperature Superconductor Thin Films at Microwave Frequencies, Springer Tracts of Modern Physics, vol. 155, Springer (Heidelberg 1999).

[3] H. Pandit, D. Shi, N. H. Babu, X. Chaud, D. A. Cardwell, P. He, D. Isfort, R. Tournier, D. Mast, and A. M. Ferendeci, Physica C 425 (2005) 44.

[4] H. Padamsee, Supercond. Sci. Technol. 14 (2001) R28.

[5] E. W. Collings, M. D. Sumption, and T. Tajima, Supercond. Sci. Technol. 17 (2004) S595.

[6] M. J. Lancaster, T. S. M. Maclean, Z. Wu, A. Porch, P. Woodall, N. NcN. Alford, IEE Proceedings-H, vol. 139 (1992) 149.

[7] Z. Zhai, C. Kusko, N. Hakim, and S. Sridhar, Rev. Sci. Instrum. 71 (2000) 3151.

[8] C. Zahopoulos, W. L. Kennedy, S. Sridhar, Appl. Phys. Lett. 52 (1988) 2168.

[9] M. Golosovsky, Particle Accelerators 351 (1998) 87, and references therein.

[10] M. R. Trunin, Physics-Uspeki 48 (2005) 979.

[11] J. Nagamatsu, N. Nakagawa, T. Muranaka, Y. Zenitani, and J. Akimitsu, Nature (London) $\mathbf{4 1 0}$ (2001) 63.

[12] Y. Bugoslavsky, G. K. Perkins, X. Qi, L. F. Cohen, and A. D. Caplin, Nature (London) 410 (2001) 563.

[13] M. A. Hein, Proceedings of URSI-GA (Maastricht 2002); e-print arXiv:cond-mat/0207226.

[14] D. C. Larbalestier, L. D. Cooley, M. O. Rikel, A.A. Polyanskii, J. Jiang, S. Patnaik, X. Y. Cai, D. M. Feldmann, A. Gurevich, A. A. Squitieri, M. T. Naus, C. B. Eom, E. E. Hellstrom, R. J. Cava, K. A. Regan, N. Rogado, M. A. Hayward, T. He, J. S. Slusky, P. Khalifah, K. Inumaru, and M. Haas, Nature (London) 410 (2001) 186.

[15] J. M. Rowell, Supercond. Sci. Technol. 16 (2003) R17.

[16] T. Tajima, Proceedings of EPAC Conf. (Paris 2002) 2289.

[17] G. Giunchi, A. Agliolo Gallitto, G. Bonsignore, M. Bonura and M. Li Vigni, Supercond. Sci. Technol. 20 (2007) L16.

[18] EDISON, patent n. MI2001A000978.

[19] G. Giunchi, Int. J. Mod. Phys. B 17 (2003) 453. 
[20] G. Giunchi, G. Ripamonti, T. Cavallin, E. Bassani, Cryogenics 46 (2006) 237.

[21] S. B. Samanta, H. Narayan, A. Gupta, A. V. Narlikar, T. Muranaka, and J. Akimtsu, Phys. Rev. B 65 (2002) 092510.

[22] Neeraj Khare, D. P. Singh, A. K. Gupta, Shashawati Sen, D. K. Aswal, S. K. Gupta, and L. C. Gupta, J. Appl. Phys. 97 (2005) 07613.

[23] A. Agliolo Gallitto, G. Bonsignore, G. Giunchi, and M. Li Vigni, J. Supercond. 20 (2007) 13.

[24] A. Agliolo Gallitto, G. Bonsignore, G. Giunchi, M. Li Vigni, and Yu. A. Nefyodov, J. Phys.: Conf. Ser. 43 (2006) 480.

[25] G. Giunchi, C. Orecchia, L. Malpezzi, and N. Masciocchi, Physica C 433 (2006) 182. 